\title{
ADVANCED CARDIAC LIFE SUPPORT COURSE - Where should we improve our learning stations? Lessons from a Portuguese Training Center
}

SANTOS, N. - Registered Nurse, Intensive Care Unit, Hospital Prof. Dr. Fernando da Fonseca EPE , Amadora, Portugal. CALDEIRA, P. - Registered Nurse, Emergency Department, Hospital de Cascais, Cascais, Portugal.

AMARAL, T. - Registered Nurse, Emergency Department, Hospital de São José, Lisboa, Portugal.

INTRODUCTION: Ocean Medical is a Portuguese International Training Center of American Heart Association for Advanced Cardiac Life Support (ACLS) since 2009. Every year about 354 medical doctors (MD) and registered nurses (RN) get through our ACLS courses, from those 29,16\% don't succeed.

AlM: $1^{\text {st }}$ - Assess the practical testing scenario checklist (PTSC) of all ACLS students between 2013 and 2017 , identifying in which algorithm students fail the most. $2^{\text {nd }}$ - Define new strategies to improve students' skills in that specific algorithm.

METHOD: An observational retrospective study was done with the analysis of all PTSC in ACLS courses since 2013 to 2016 . From a total of 1415 ACLS students, 177 were excluded due to incomplete data. Every student had 2 opportunities to successfully complete this task. For those who didn't make it at first we considered for analysis the second one. For each student we've outlined the algorithm that leads to failure. It was considered statistically significant a $p$-value $<0.05$.

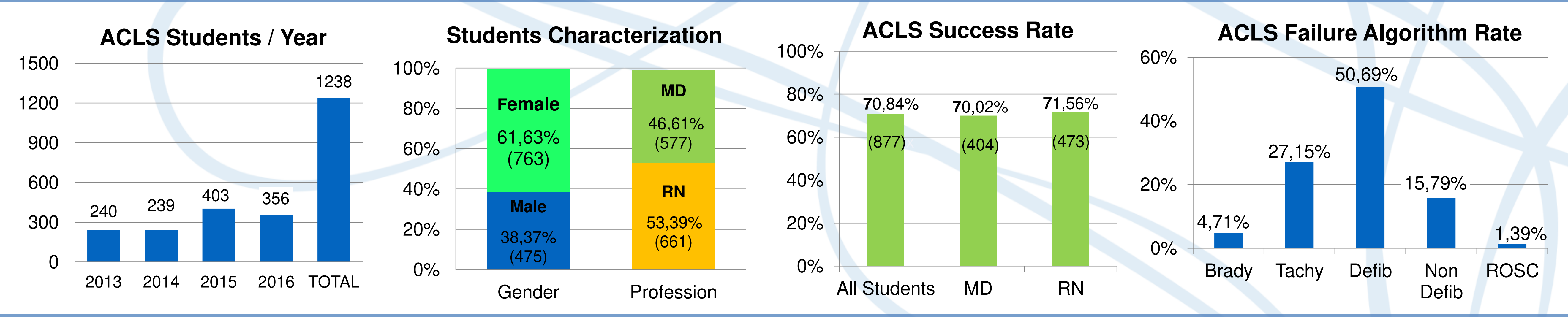

LIMITATIONS: The actual PTSC used in ACLS course does note allow us to accurate describe the student error.

CONCLUSIONS: Most students who failed ACLS course did it at cardiac arrest algorithm, on defibrillation rhythms, followed by the tachycardia algorithm. We have also verified that doctors and nurses have very similar successful ratios $(70,02 \%$ VS $71,56 \% ; p<0,001)$. Increasing training time in these algorithms - theoretical learning stations and technical skills training - can be a solution. We need to improve the evaluation report so that it will be possible to isolate the technical and non-technical skills deficits and thus be able to implement more direct measures to increase the ACLS course learning rate. 